\title{
Preconditioning, induced by sub-toxic dose of the neurotoxin L-BMAA, delays ALS progression in mice and prevents $\mathrm{Na}^{+} / \mathrm{Ca}^{2+}$ exchanger 3 downregulation
}

Serenella Anzilotti ${ }^{1}$, Paola Brancaccio², Giuseppe Simeone ${ }^{2}$, Valeria Valsecchi², Antonio Vinciguerra², Agnese Secondo $\mathbb{0}^{2}$, Tiziana Petrozziello², Natascia Guida', Rossana Sirabella², Ornella Cuomo², Pasquale Cepparulo², Andrè Herchuelz ${ }^{3}$, Salvatore Amoroso ${ }^{2,4}$, Gianfranco Di Renzo ${ }^{2}$, Lucio Annunziato ${ }^{1,2}$ and Giuseppe Pignataro (i) ${ }^{2}$

\begin{abstract}
Preconditioning $(\mathrm{PC})$ is a phenomenon wherein a mild insult induces resistance to a later, severe injury. Although PC has been extensively studied in several neurological disorders, no studies have been performed in amyotrophic lateral sclerosis (ALS). Here we hypothesize that a sub-toxic acute exposure to the cycad neurotoxin beta-methylamino-Lalanine (L-BMAA) is able to delay ALS progression in SOD1 G93A mice and that NCX3, a membrane transporter able to handle the deregulation of ionic homeostasis occurring during ALS, takes part to this neuroprotective effect.

Preconditioning effect was examined on disease onset and duration, motor functions, and motor neurons in terms of functional declines and severity of histological damage in male and female mice. Our findings demonstrate that a subtoxic dose of L-BMAA works as preconditioning stimulus and is able to delay ALS onset and to prolong ALS mice survival. Interestingly, preconditioning prevented NCX3 downregulation in SOD1 G93A mice spinal cord, leading to an increased number of motor neurons associated to a reduced astrogliosis, and reduced the denervation of neuromuscular junctions observed in SOD1 G93A mice. These protective effects were mitigated in ncx3+/- mice. This study established for the first time an animal model of preconditioning in ALS and candidates NCX3 as a new therapeutic target.
\end{abstract}

\section{Introduction}

Preconditioning $(\mathrm{PC})$ is a phenomenon wherein a mild insult induces a cellular and tissue resistance to a later severe injury ${ }^{1}$.

Over the years numerous stimuli were described as possible PC inductors. Among these, hypoxic stimuli, bacterial toxins such as LPS, small seizures, volatile anesthetics, hyperthermia, and hypothermia ${ }^{1}$. Protection

\footnotetext{
Correspondence: Giuseppe Pignataro (giuseppe.pignataro@unina.it) ${ }^{1}$ IRCCS SDN, Via Gianturco, 80131 Naples, Italy

2Division of Pharmacology, Department Neuroscience, School of Medicine, Federico II University of Naples, Via Pansini, 5, 80131 Naples, Italy

Full list of author information is available at the end of the article

Edited by A. Verkhratsky
}

triggered by these stimuli is usually divided by a temporal point of view in acute, rapid PC, and long-lasting, delayed $\mathrm{PC}^{1}$. In particular, the delayed $\mathrm{PC}$ includes the involvement of a genomic reprogramming, which wavers, in most cases, in a downregulation or upregulation of proteins involved in the pathogenesis of the disease ${ }^{2}$. To date, although PC has been extensively studied in several neurological disorders such as Parkinson disease, brain ischemia, and epilepsy, no evidence has been provided on the existence of this PC neuroprotection strategy in amyotrophic lateral sclerosis (ALS).

The aim of this study was therefore to identify candidate stimuli and/or genes that can activate the 
pro-regenerative preconditioned state and to characterize the first PC model in ALS.

To this aim, we hypothesized that a sub-toxic dose of the cycad neurotoxin L-BMAA, an excitatory non-protein amino acid produced by cyanobacteria and associated with amyotrophic lateral sclerosis-Parkinson dementia complex (ALS-PDC), in Guam indigenous population, could be used as a PC stimulus. It is well known, in fact, that the toxin L-BMAA represents a possible cause of ALS linked to environmental factors. In fact, people who chronically feed foods rich in L-BMAA seem to contract the disease more frequently ${ }^{3-5}$. These data are supported by in vitro and in vivo experimental studies showing that this toxin may elicit ALS features ${ }^{3-5}$.

On this basis and assuming that a sub-toxic dose of the toxin could serve as a PC stimulus, experiments were conducted to verify: (a) whether the supposed PC stimulus could act as neuroprotectant, and (b) to elucidate the mechanisms underlying this phenomenon. Among the proposed putative mechanisms, it has been investigated the role of a plasma membrane ionic transporter, namely $\mathrm{Na}^{+} / \mathrm{Ca}^{2+}$ exchanger (NCX). Indeed, it is well known that alteration of calcium homeostasis is of significant importance in the pathogenesis of $\mathrm{ALS}^{6,7}$. In fact, in recent years it has been shown that the selective vulnerability of motor neurons in ALS may be due to the reduced capacity of these cells to buffer the excess of calcium ions that occurs in the course of the disease ${ }^{7}$. This concept arises from several studies carried out in humans and in cellular and experimental animal models of ALS in which $\mathrm{Ca}^{2+}$ binding proteins such as calbindin are reduced during the progression of the disease ${ }^{8}$. These findings are in good agreement with the demonstration that in patients and ALS mice, in those regions that precociously undergo to degeneration, such as facial, spinal, and hypoglossal motor neurons, the neuronal cytosolic $\mathrm{Ca}^{2+}$ buffering capacity is lower, whereas those areas

that are more resistant to the disease display a greater cytosolic $\mathrm{Ca}^{2+}$ buffering capacity ${ }^{9}$. As NCX is one of the main mechanisms by which calcium and sodium ions can be extruded from the cell, we decided to investigate its role.

NCX is a membrane transporter that, by regulating the homeostasis of $\mathrm{Na}^{+}$and $\mathrm{Ca}^{2+}$, participates to the evolution of several neurological disorders including brain ischemia, epilepsy, multiple sclerosis and Alzheimer disease $\mathrm{e}^{10-15}$. To date three different isoforms, NCX1-3, and several splicing variants have been described within the CNS. The specific role of each isoform in ALS pathophysiology has not yet been determined, nevertheless some seminal works attribute to NCX3 a pivotal role in neuromuscular transmission impairment ${ }^{16-18}$. These information render this transporter particularly interesting as putative druggable target in ALS.

\section{RESULTS}

NCX3 transcript and protein expression decreased in spinal cord, brain stem, and gastrocnemius muscle of SOD1 G93A mice

To examine the role of NCX3 in ALS, we first determined NCX3 expression levels in affected CNS regions and in the gastrocnemius muscle of WT and G93A mice by real-time PCR and western blot analysis.

In particular, at 2 months of age, when SOD1 G93A mice were still asymptomatic, the expression of NCX3 mRNA appeared strongly reduced (50\%) only in the spinal cord (Fig. 1c). Although, at 4.5 months, when SOD1 G93A mice were fully symptomatic, NCX3 mRNA expression was also reduced in brain stem area, spinal cord, and gastrocnemius (Fig. 1b-d). No changes in mRNA NCX3 levels were observed in motor cortex area in the asymptomatic and symptomatic phases (Fig. 1a).

The expression of NCX3 protein appeared significantly reduced in spinal cord, brain stem and gastrocnemius of SOD1 G93A mice in the fully symptomatic phase, 4.5 months (Fig. 1f-h) and in the brain stem and spinal cord of pre-symptomatic animals, 2 months. No changes were observed in motor cortex area (Fig. 1e).

\section{PC induced by sub-toxic treatment with the cycad toxin L- BMAA prevented NCX3 expression and activity downregulation in spinal cord and brain stem of SOD1 G93A mice}

To prevent NCX3 decrease observed in spinal cord and brain stem areas of SOD1 G93A mice, we settled up a PC protocol by an acute intracerebroventricular injection of L-BMAA toxin $(4.5 \mathrm{mM} / 1 \mu \mathrm{l})$ and then, we evaluated NCX3 activity and expression by microfluorimetry, immunohistochemical, and western blotting analysis. SOD1 G93A mice treated with a sub-toxic dose of LBMAA or saline, and a comparison group of wild-type mice treated with a sub-toxic dose of L-BMAA or vehicle, were killed 7 days after L-BMAA injection. To verify the effect of L-BMAA-induced PC on NCX3 activity, NCX reverse mode was evaluated by $\mathrm{Na}^{+}$-free-induced $\left[\mathrm{Ca}^{2+}\right]_{\mathrm{i}}$ increase in spinal cord synaptosomal preparations from $\mathrm{L}$ BMAA-treated SOD1 G93A mice, vehicle-treated SOD1 G93A mice, and vehicle-treated SOD1 wild-type mice, all at 4.5 months. $\mathrm{Na}^{+}$-free-induced $\left[\mathrm{Ca}^{2+}\right]_{\mathrm{i}}$ increase was significantly reduced in synaptosomes from vehicletreated SOD1 G93A mice compared to wild-type animals. Interestingly, L-BMAA PC significantly prevented the reduction of NCX activity registered in synaptosomes from SOD1 G93A mice (Fig. 2a).

Accordingly, further investigations by confocal microscopy experiments indicated that NCX3 expression 


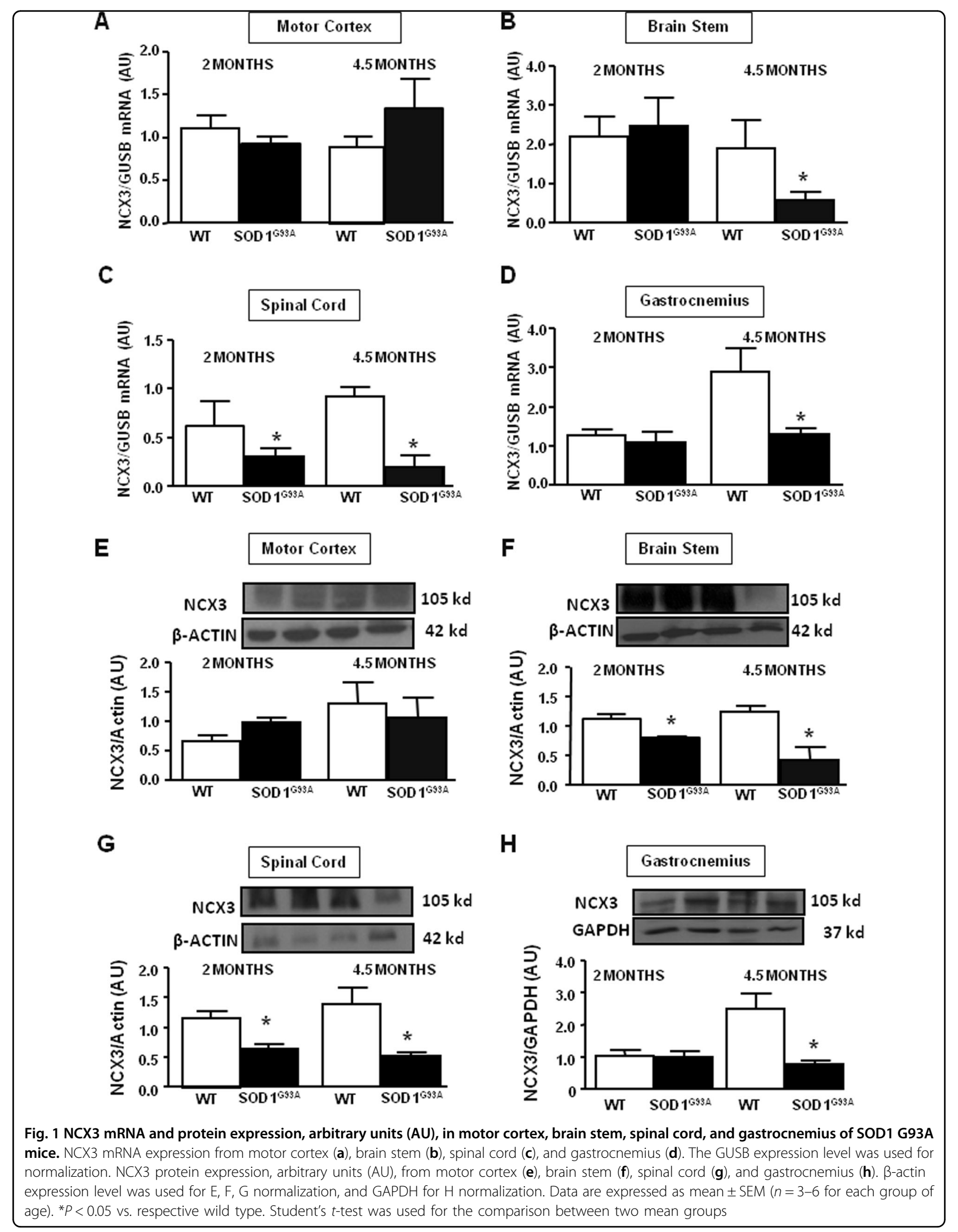




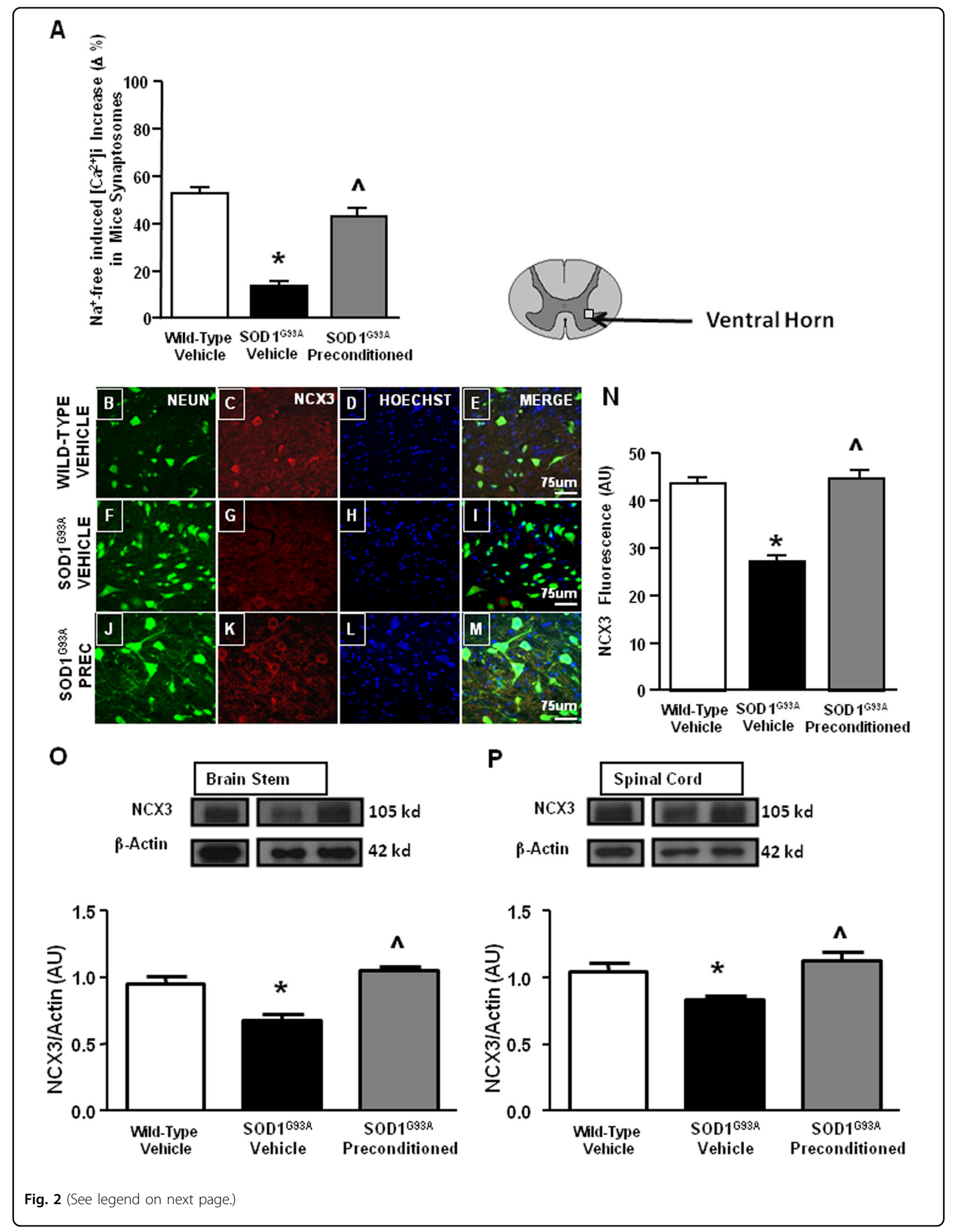


(see figure on previous page)

Fig. 2 NCX3 immunolocalization, expression, and quantification of total NCX activity in SOD1 G93A mice subjected to L-BMAA-induced PC. Quantification of NCX activity as $\left[\mathrm{Ca}^{2+}\right]$ i increase induced by $\mathrm{Na}^{+}$-free perfusion in Fura-2 AM-loaded spinal cord synaptosomes of adult wild-type mice treated with vehicle and symptomatic SOD1 G93A mice treated with vehicle or preconditioned with L-BMAA. ${ }^{*} P<0.05$ vs. wild-type vehicle; ${ }^{\wedge} P$ $<0.05$ vs. SOD1 G93A mice (a). Double labeling of NCX3 and NeuN in spinal cord of pre-symptomatic wild-type mice (b-e), SOD1 G93A mice treated with vehicle (f-i) and SOD1 G93A mice treated with L-BMAA PC (j-m). Scale bar $75 \mu \mathrm{m}$. Quantification of NCX3 fluorescence as arbitrary units (AU). *P $<0.05$ vs. wild-type vehicle; $\wedge P<0.05$ vs. SOD1 G93A mice (n). Representative Western blotting and quantification of the effect of L-BMAA-induced PC on NCX3 protein expression, arbitrary units (AU), in brain stem (o) and spinal cord (p). ${ }^{*} P<0.05$ vs. wild-type vehicle; $\wedge P<0.05$ vs. SOD1 G93A mice. The $\beta$-actin expression level was used for normalization. Data are expressed as mean \pm SEM $\left(n=5-6\right.$ for each group). ${ }^{*} P<0.05$ vs. wild-type vehicle and SOD1 G93A preconditioned mice. $P$ values were obtained using one-way ANOVA with Newman Keuls's correction for multiple comparisons

increased in L-BMAA preconditioned SOD1 G93A compared to vehicle-treated SOD1 G93A mice (Fig. 2b-n). Interestingly, although NCX3 was present in all neuronal populations, its expression was prevalent in large polygonal-shaped neurons, such as motor neurons (Fig. 2b-m).

Finally, western blotting experiments confirmed that L-BMAA PC determined an upregulation of NCX3 expression in spinal cord and brain stem areas. In fact, in these two CNS regions, NCX3 protein expression was significantly higher in L-BMAA preconditioned SOD1 G93A mice than in vehicle-treated SOD1 G93A mice (Fig. 2o-p).

No effect on NCX activity and NCX3 expression were observed when wild-type animals were treated with subtoxic dose of L-BMAA (Data not shown).

Notably, although NCX2 expression was reduced during ALS progression, $\mathrm{PC}$ was not able to induce any effect on NCX1 and NCX2 expression evaluated in brain stem and motor cortex of SOD1 G93A mice (Fig. S1).

\section{PC prevented motor neuron degeneration in ventral horn and nucleus facialis of SOD1 G93A mice}

To further demonstrate that the protective effect of PC is correlated to NCX3, we generated SOD1 G93A/ncx3 $+/-$ mice by crossing SOD1 G93A mice with $n c x 3+/-$ mice.

Sections of the spinal cord and brain stem nucleus facialis of these mice at the fully symptomatic phase of disease were stained with Nissl, and motor neurons were counted in the defined spinal cord and brain stem regions of interest. As ALS degeneration occurs preferentially in large motor neurons, the number of motor neurons with a perikaryal projection area of more than $200 \mu \mathrm{m}^{2}$ was counted. PC by L-BMAA treatment preserved large motor neurons in the spinal cord (number of motorneuros per square $\mathrm{mm}$ in wild-type animals $292 \pm 15$; G93A mice $150 \pm 3$; G93A preconditioned mice $228 \pm 2$; G93A/ncx3 $+/-$ mice $136 \pm 5$; and G93A/ncx3+/- preconditioned mice $141 \pm 7)$ and in the brain stem facialis nucleus (numer of motorneuros per square $\mathrm{mm}$ in wild-type animals $1800 \pm 9 ; \quad$ G93A mice $792 \pm 3 ; \quad$ G93A preconditioned mice $1442 \pm 5$; G93A/ncx3+/ - mice 696 \pm 9 ; and $\mathrm{G} 93 \mathrm{~A} / \mathrm{ncx} 3+/$ - preconditioned mice $793 \pm 12$ ) compared to vehicle-treated SOD1 G93A mice (Fig. 3a-d). Interestingly, PC failed to prevent the loss of motor neurons when applied to SOD1 G93A/ncx3+/mice, thus suggesting that NCX3 contributes to the protection mediated by L-BMAA-induced PC (Fig. 3a-d).

\section{L-BMAA-induced PC reduced astroglia but not microglia proliferation in the spinal cord of SOD1 G93A mice}

The motor neuron damage caused by expression of mutant human SOD1 induces the activation of astroglia and microglia, evaluated as GFAP and IBA-1 expression (Fig. 4). Interestingly, PC was able to prevent GFAP overexpression but not IBA-1 overexpression, thus suggesting that the reduced astrogliosis contributed to PCinduced protection. Indeed, GFAP-immunoreactive astrocytes were more abundant in vehicle-treated SOD1 G93A mice compared to preconditioned SOD1 G93A mice (Fig. 4i-p). Notably, when PC was induced in SOD1 G93A/ ncx3+/- mice, astrogliosis was no longer reduced (Fig. 5).

\section{L-BMAA-induced PC increased fully innervated end plates in neuromuscular junctions (NMJ) of SOD1 G93A mice}

We first examined the effect of PC on NMJ innervation in 4.5 months old wild-type, G93A mice and G93A/ncx3 +/ - mice by assessing co-localization of fluorescently labeled pre-synaptic and post-synaptic NMJ markers in the gastrocnemius muscle (Fig. 6a-o). NMJs were classed as "fully innervated", "partially innervated", or "denervated". As expected, by late-stage disease, a large proportion of NMJs exhibited complete $(75.7 \pm 7.0 \%)$ or partial $(21.6 \pm 2.4 \%)$ denervation in G93A mice, while only $2.5 \pm 0.7 \%$ remained fully innervated. However, PC resulted in a significant increase in the proportion of fully innervated NMJs $(15.4 \pm 2.0 \%)$ and an equally marked decrease in the proportion of denervated NM)s $(35.0 \pm$ 7.0\%) (Fig. 6p).

Notably, PC was not able to exert its protective effect in G93A animals crossed with ncx $3+/-$ mice (\% of denerveted end plates $76.3 \pm 8.8$ ) (Fig. $6 \mathrm{p}$ ), thus indicating that 


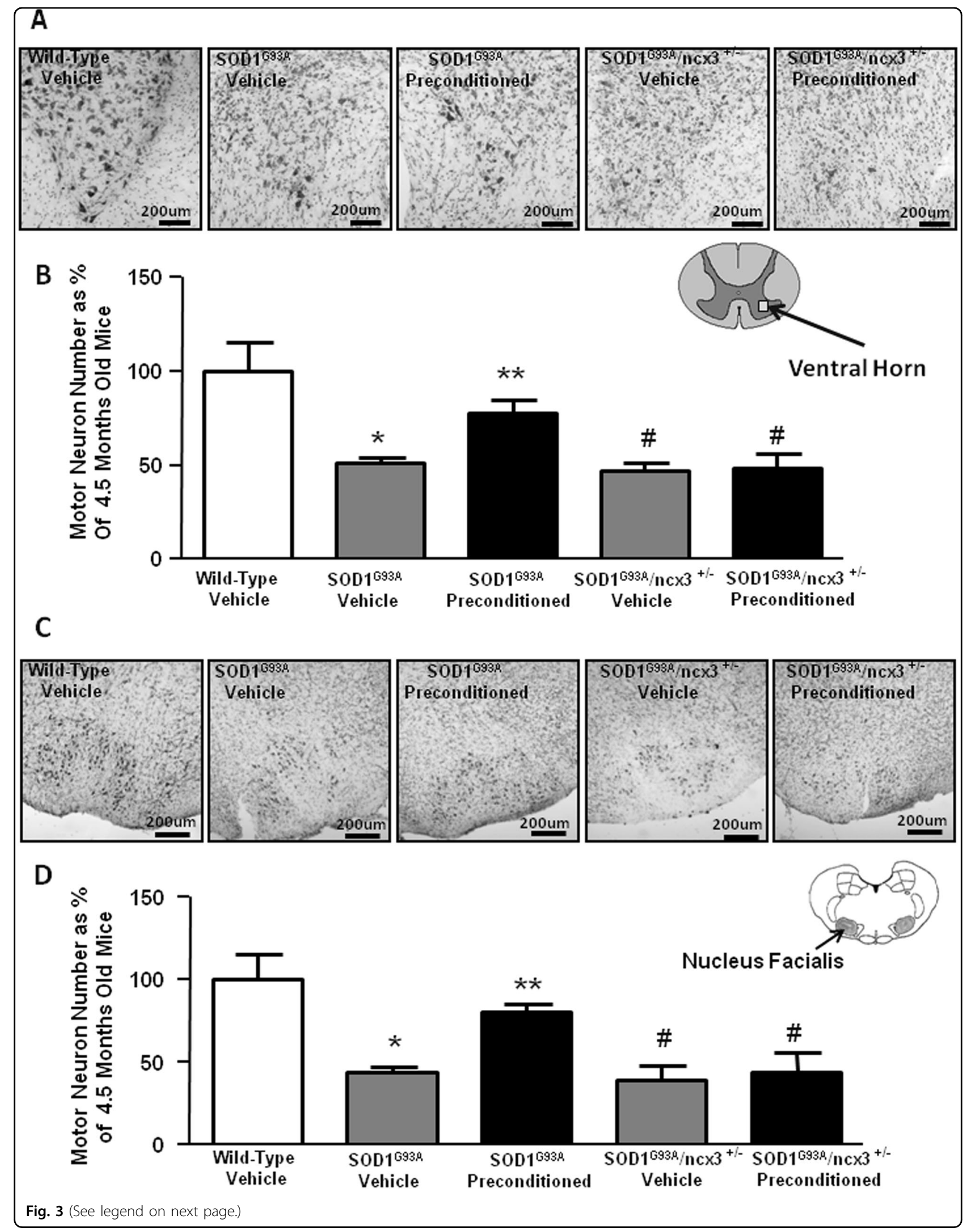


(see figure on previous page)

Fig. 3 Effect of L-BMAA-induced PC on motor neurons survival. Representative image of Nissl stained in spinal cord (a). Scale bar $200 \mu \mathrm{m}$. Cell counting analysis of motor neurons expressed as the percentage of total motor neurons in spinal cord of 4.5 month-old SOD1 G93A mice treated with vehicle or L-BMAA compared to wild-type vehicle (b). Representative image of Nissl stained in nucleus facialis (c). Scale bar $200 \mu \mathrm{m}$. Cell counting analysis of motor neurons expressed as the percentage of total motor neurons in nucleus facialis of 4.5 month-old SOD1 G93A mice treated with vehicle or L-BMAA compared to wild-type vehicle (d). Data are expressed as mean \pm SEM ( $n=4$ for each group). ${ }^{*} P<0.05$ vs. wild-type vehicle ${ }^{* *} P<$ 0.05 vs. all other experimental groups. $P$ values were obtained using one-way ANOVA with Newman Keuls's correction for multiple comparisons

the presence of NCX3 is necessary to mediate this protective effect.

\section{L-BMAA-induced PC improved survival rate and motor performances, delayed paralysis onset, and prevented body weight loss in SOD1 G93A mice during the development of the disease}

L-BMAA PC prolonged the survival of SOD1 G93A mice compared to vehicle-treated animals $(123.3 \pm 3$ vs. $142.5 \pm 4$ survival average days) (Fig. 7Aa, b). By contrast, L-BMAA PC was not able to prolong mice survival in SOD1 G93A/ ncx $3+/-$ mice, $(119 \pm 6$ vs. $123 \pm 4$ survival average days) thus showing that NCX3 contributes to PC effect (Fig. 8Aa, b).

The important role of NCX3 in mediating PC protection was confirmed by examining other parameters of ALS progression such as paralysis onset, body weight (Figs. 7b and 8b) rotarod (Figs. 7c and 8c) and grip performance (Figs. $7 \mathrm{~d}$ and $8 \mathrm{~d}$ ). Indeed, the protective effect of $\mathrm{PC}$ was absent in SOD1 G93A/ ncx3+/- mice.

In fact, L-BMAA PC prevented the body weight loss observed in vehicle-treated SOD1 G93A mice from 7 weeks to 9 weeks after treatment (Fig. 7Bc). Conversely, in SOD1 G93A/ ncx3+/- mice, PC did not modify this parameter (Fig. 8Bc).

In a similar way, L-BMAA PC delayed the onset of disease by 14 days $(46.4 \pm 3$ vs. $59.7 \pm 5$ days) in SOD1 G93A mice. By contrast, L-BMAA PC failed to delay paralysis onset in SOD1 G93A/ ncx3+/- mice (paralysis onset: $46 \pm 3$ days in SOD1 G93A mice; $60 \pm 5$ days in preconditioned SOD1 G93A mice; $44 \pm 4$ in SOD1 G93A/ ncx $3+/-$ mice and $43 \pm 5$ days in preconditioned SOD1 G93A/ ncx3+/- mice) (Figs. 7Ba, b and 8Ba, b).

In addition, rotarod test showed that L-BMAA PC increased time spent on rotarod in SOD1 G93A mice but not in SOD1 G93A/ ncx3+/- mice (Figs. 7C and 8C).

Moreover, L-BMAA PC was able to attenuate the decline in grip performance in SOD1 G93A mice but not in SOD1 G93A/ ncx3+/- mice (Figs. 7D and 8D).

\section{DISCUSSION}

The present study established for the first time an animal model for studying the possible protective role exerted by PC in SOD1 G93A mice based on the administration of a sub-toxic dose of the cycad neurotoxin L-BMAA. In addition, we demonstrated (a) that the cycad toxin L-BMAA, at this sub-toxic dose, improves survival rate and motor performances, delays paralysis onset and prevents body weight loss in SOD1 G93A mice during the development of the disease and that (b) the plasma membrane transporter $\mathrm{Na}^{+} / \mathrm{Ca}^{2+}$ exchanger 3 , NCX3, contributes to the protection elicited by L-BMAAPC thus representing a target for setting on new strategies in ALS intervention.

PC is a phenomenon wherein a mild insult induces a cellular and tissue resistance to a later severe injury ${ }^{1,19}$. Many different stimuli, named triggers, such as chemicals, ischemia, hypoxia, and hypothermia lead to PC. "Triggers" generate "transducers" and "effectors", such as chemical mediators, neurotransmitters, and proteins, that elicit PCprotection ${ }^{1}$. In experimental models, protection through PC has been consistently demonstrated across multiple organ systems and in many different animal species, leaving no doubt on the existence of this phenomenon. For instance, in ischemic PC exposing an organ to brief ischemia induces temporary resistance to more severe ischemia, in the same or even a distant organ, i.e., remote conditioning ${ }^{1,20-23}$.

That a protein involved in calcium and sodium homeostasis maintenance could be linked to ALS pathophysiology was clearly conceivable from previous reports ${ }^{6,24,25}$. In fact, recent evidence suggest that abnormalities in cellular $\mathrm{Ca}^{2+}$ signaling are common features in the pathogenesis of a range of neurodegenerative disorders, including ALS ${ }^{6,24,25}$. It is well known that $\mathrm{Ca}^{2+}$ is one of the most relevant intracellular messengers, being essential in neuronal development, synaptic transmission and plasticity, as well as in the regulation of various transduction pathways in the brain ${ }^{7}$. In subtypes of ALS associated with the SOD1 mutation and in the sporadic disease, there have been several reports indicating the involvement of intracellular $\mathrm{Ca}^{2+}$ homeostasis disruption in ALS pathophysiology $6,7,24,25$. Combining conclusions from multiple animal models as well as cell culture models used to determine pathogenic mechanisms in ALS, the central insight is that selective vulnerability of MNs likely arises from a combination of several mechanisms; two of them, such as mitochondrial 


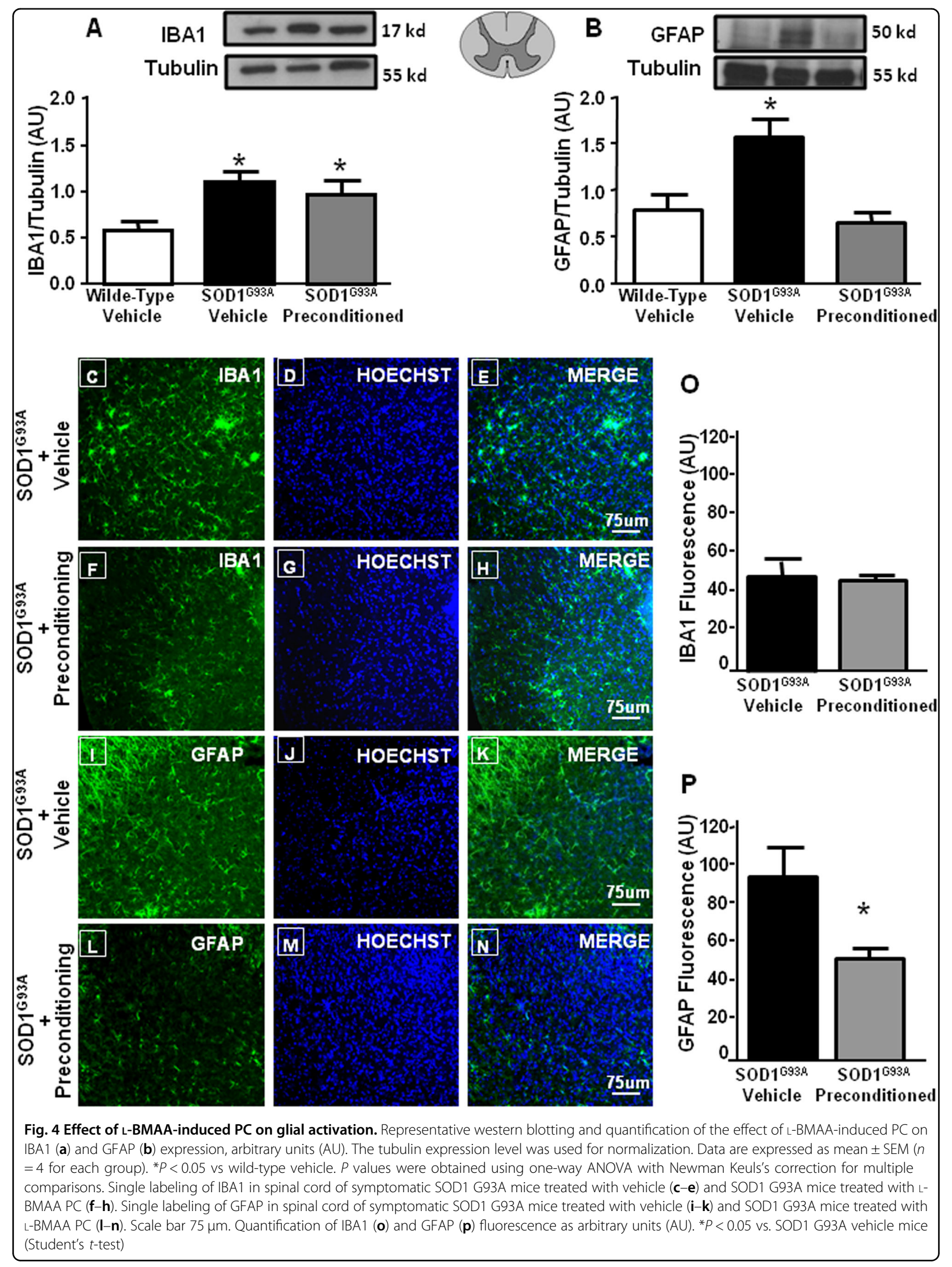




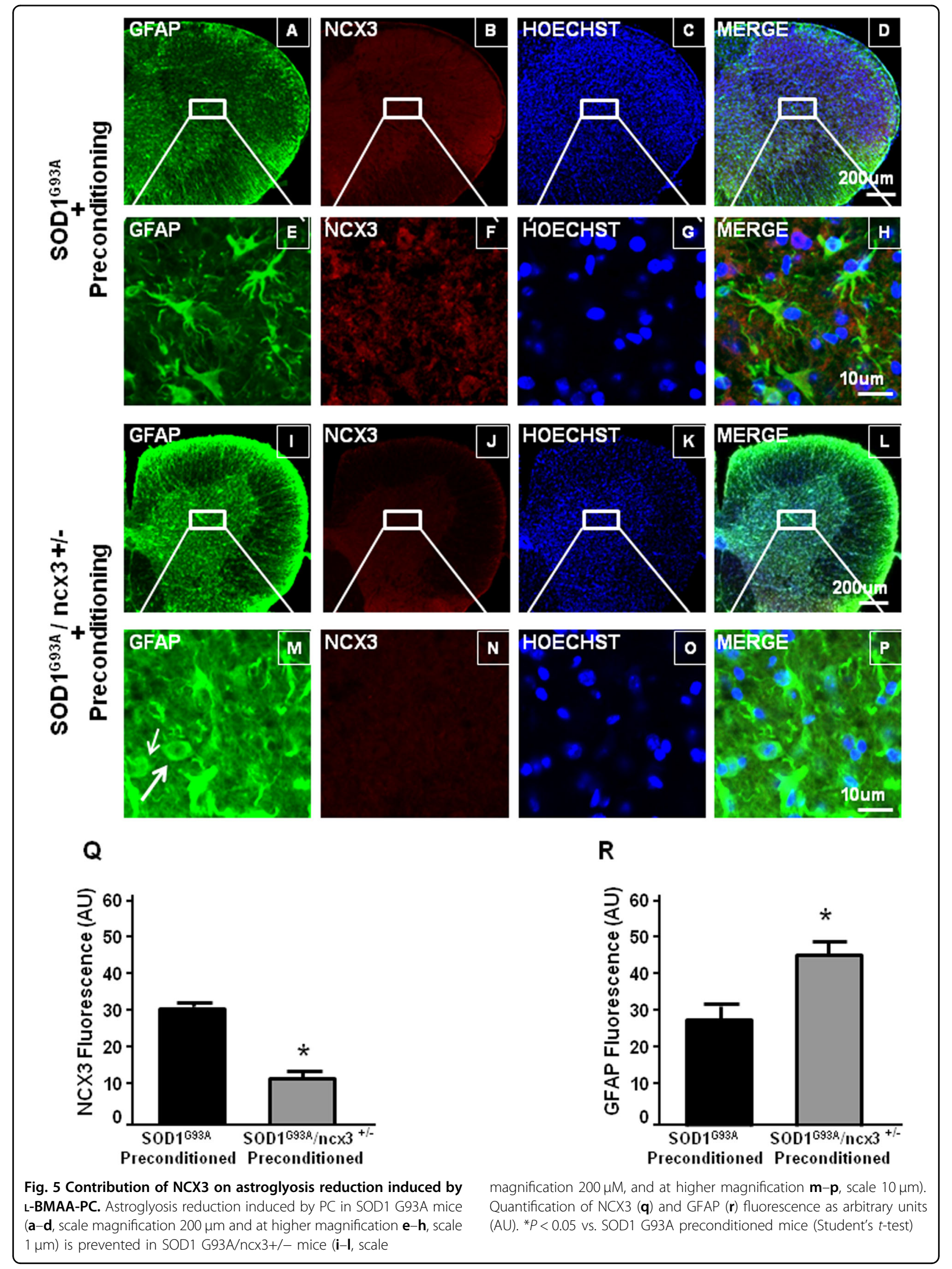




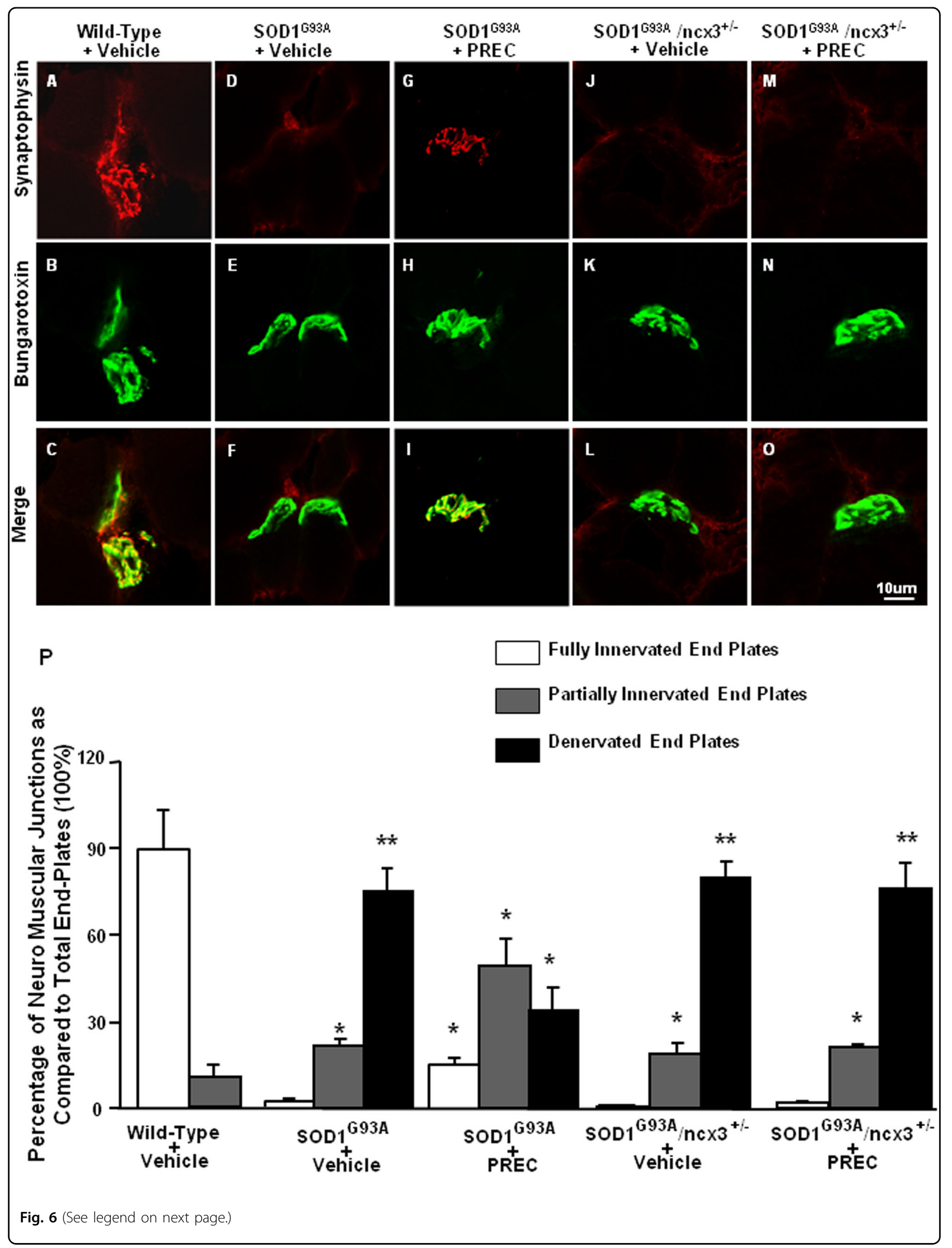


(see figure on previous page)

Fig. 6 Effect of L-BMAA-induced PC on NMJs innervations of SOD1 G93A mice. Double labeling of synaptophysin and bungarotoxin of symptomatic wild-type mice treated with vehicle $(\mathbf{a}-\mathbf{c})$, SOD1 G93A mice treated with vehicle $(\mathbf{d}-\mathbf{f})$, SOD1 G93A mice treated with L-BMAA PC ( $(\mathbf{g}-\mathbf{i})$, SOD1 G93A/ncx3+/- mice treated with vehicle $(\mathbf{j}-\mathbf{l})$ and SOD1 G93A /ncx3+/- mice treated with L-BMAA PC (m-o). Scale bar $10 \mu \mathrm{m}$. Cell counting analysis of gastrocnemius NMJs of wild-type vehicle, SOD1 G93A vehicle, SOD1 G93A PC, SOD1 G93A/ncx3+/- vehicle and SOD1 G93A /ncx3+/- PC. The data are expressed as the percentage of 270 or more NMJs from each group. Data are expressed as mean \pm SEM $\left(n=5\right.$ for each group). ${ }^{*} P<0.05$ vs. respective wild-type vehicle. ${ }^{* *} P<0.05$ vs. all other experimental groups. $P$ values were obtained using two-way ANOVA with Bonferroni's correction for multiple comparisons

dysfunction and $\mathrm{Ca}^{2+}$ homeostasis are prominent ${ }^{25}$. By documenting the involvement and relevance of an alteration of $\mathrm{Ca}^{2+}$ homeostasis in ALS pathophysiology, it is possible to propose two scenarios: the first one is that motor neurons possess large number of transporters and ionic channels that, when activated, cause rapid $\mathrm{Ca}^{2+}$ influx, which, in part because of relatively weak cytosolic $\mathrm{Ca}^{2+}$ buffering, results in mitochondrial $\mathrm{Ca}^{2+}$ overload and strong ROS generation. In addition, it is possible to hypothesize that, in ALS affected tissues, the extrusion mechanisms, mainly represented by the high capacity-low affinity plasma membrane $\mathrm{Na}^{+} / \mathrm{Ca}^{2+}$ exchanger, are impaired and cannot provide an efficient removal of calcium ions. Results of the present study support this hypothesis. In fact, in line with these premises, we documented a reduction in NCX3 expression in motor neurons and muscle of asymptomatic G93A mice. Differently from NCX1 and NCX2, the other two CNS isoforms, the reduction in NCX3 expression was mitigated by $\mathrm{PC}$ treatment thus underlining the important role of NCX3 in ALS pathophysiology. In fact, in asymptomatic SOD1 G93A mice, PC treatment with L-BMAA did not modify the expression of NCX1 and NCX2 (data not shown). It should be underlined that NCX3 is not the only protein controlling intracellular $\mathrm{Ca}^{2+}$ concentration that is downregulated during ALS progression (see Supplemental Figure 1). In fact, we demonstrated that NCX3 is the only protein whose downregulation induced by ALS is prevented by PC. The effect on intra-synaptosomal $\mathrm{Ca}^{2+}$ reduction can, therefore, be ascribed, also to other proteins controlling intracellular $\mathrm{Ca}^{2+}$ concentration such as NCX2. The reduction of NCX3 protein expression was functionally mirrored by the reduction of the exchanger activity in synaptosomal preparations obtained from brain stem of SOD1 G93A mice. More importantly, in ncx3+/mice the protection elicited by L-BMAA-induced PC was almost completely prevented, thus underlining the importance of this transporter in mediating PC-induced protection in ALS. Indeed, the increased NCX3 expression induced by $\mathrm{PC}$ is partially prevented in ncx3+/mice, this is sufficient to prevent $\mathrm{PC}$-induced protection. The fact that NCX3 may represent one of the effector of ALS PC is in line with previous works demonstrating that the genetic ablation of this transporter worsens the course of several neurological disorders such as brain ischemia, multiple sclerosis, Alzheimer disease and epilepsy ${ }^{12,13}$.

In conclusion, this study candidates NCX3 as a putative target in the strategy for alleviating ALS. The pharmacological activation or the overexpression of NCX3, by reducing the alteration in ionic homeostasis occurring in ALS, can mitigate motor neurons degeneration in ALS.

\section{MATERIALS AND METHODS}

\section{Animal model}

B6SJL-TgN SOD1/G93A(+)1Gur mice expressing high copy number of mutant human SOD1 with a Gly93Ala substitution [SOD1 G93A] and B6SJL-TgN (SOD1)2Gur mice expressing wild-type human SOD1 (WT) ${ }^{26}$ were obtained from Jackson Laboratories (Bar Harbor, ME, USA). Transgenic animals have been crossed with background-matched B6SJL wild-type female and selective breeding maintained each transgene in the hemizygous state. All transgenic mice were identified analyzing extracts from tail tips by staining for SOD1 as previously described $^{27}$. To generate double-mutants carrying the NCX3+/ - heterozygous mutation and the SOD1 G93A transgene (SOD1 G93A;ncx3+/-), SOD1 G93A male mice (mixed C57BL6-SJL background) were bred with ncx3+/- females (Sv129 background). NCX3 knockout mice (ncx3-/-) were generated by our research group as previously described $^{16}$.

Overall, 120 male and female mice (50\% each gender) housed under diurnal lighting conditions ( $12 \mathrm{~h}$ darkness/ light) were used, 13 male and 12 female out of 120 animals were not included in the experimental groups as they died for unknown reasons. The number of female and male mice was balanced among all the experimental groups. Animals excluded were equally distributed among the experimental groups.

Experiments were performed according to the international guidelines for animal research and approved by the Animal Care Committee of "Federico II" University of Naples, Italy and Ministry of Health, Italy. All efforts were made to minimize animal suffering and to reduce the number of animals used. 


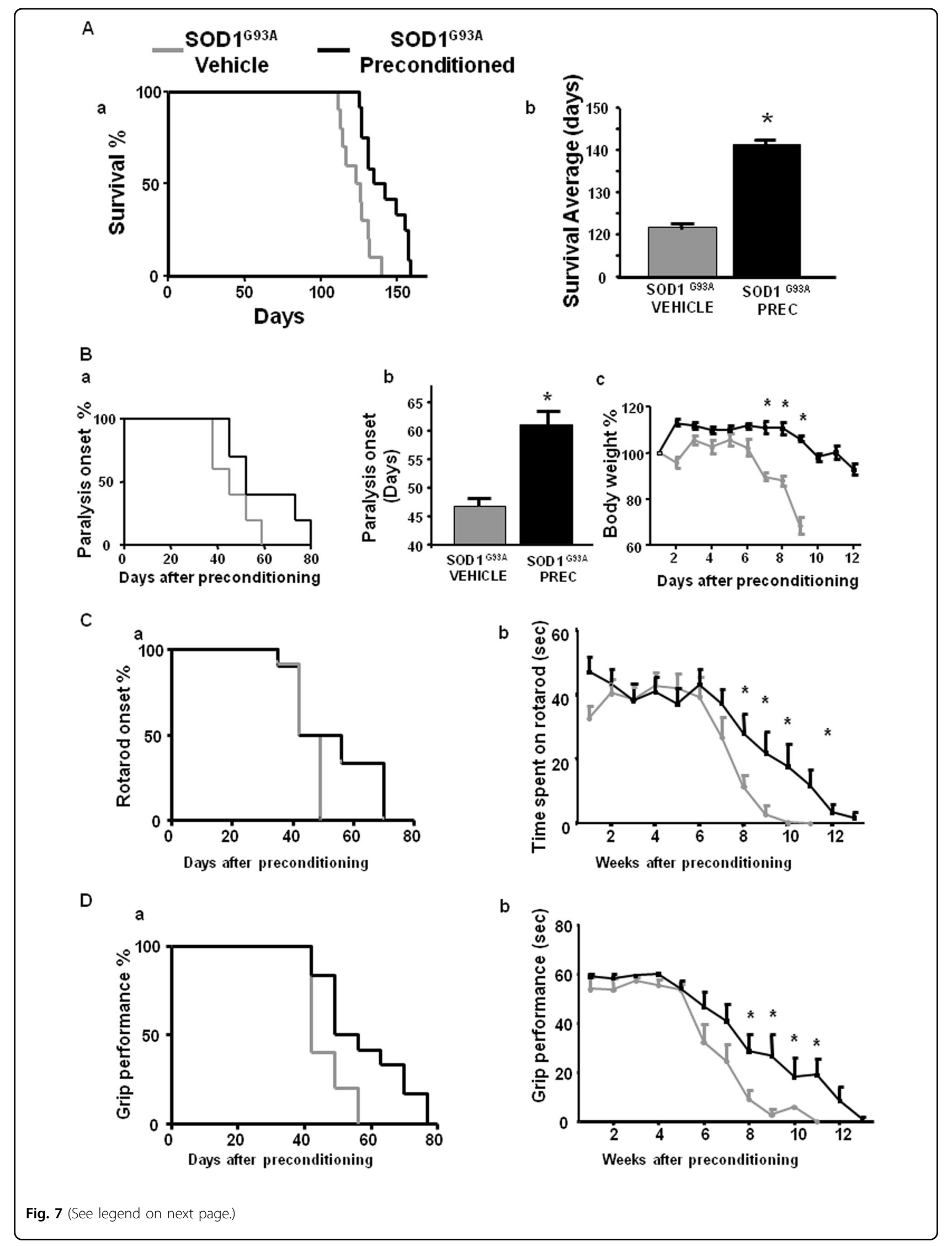


(see figure on previous page)

Fig. 7 Effect of L-BMAA-induced PC on survival, paralysis onset, body weight reduction, and motor functions in SOD1 G93A mice. Survival curve of SOD1 G93A mice treated with vehicle compared to L-BMAA preconditioned SOD1 G93A mice (Aa, b). Survival is expressed as percentage (Aa) or in days (Ab). Paralysis onset of SOD1 G93A mice treated with vehicle compared to L-BMAA preconditioned SOD1 G93A mice (Ba, b). Paralysis onset is expressed in days after treatment with vehicle or L-BMAA PC (Ba) or as percentage (Bb). Percentage of body weight reduction in SOD1 G93A vehicle compared to SOD1 G93A mice preconditioned with L-BMAA $(\mathbf{B} C)$. Percentage of rotarod onset and time spent on rotarod by SOD1 G93A mice treated with vehicle compared to SOD1 G93A mice preconditioned with L-BMAA (Ca, b). Grip performance of SOD1 G93A mice treated with vehicle compared to SOD1 G93A mice preconditioned with L-BMAA expressed as percentage (Da) or in seconds (Db). Data are expressed as mean \pm SEM for $\mathbf{A}, b ; \mathbf{B}, b, c ; C, b ;$ and $\mathbf{D}, b . n=10$ for SOD1 G93A mice treated with vehicle, $n=12$ for SOD1 G93A mice preconditioned with $L-B M A A$, ${ }^{*} P<0.05$ vs. SOD1 G93A vehicle mice. Kaplan-Meier plot was used for $\mathbf{A} a, \mathbf{B} a, \mathbf{C} a$, and $\mathbf{D a}$. Student's t-test was used for $\mathbf{A b}, \mathbf{B b}, \mathbf{C}, \mathbf{C b}, \mathbf{D b}$

\section{L-BMAA-induced PC procedure}

L-BMAA was dissolved in saline solution $(4.5 \mathrm{mM})$ and $1 \mu \mathrm{l}$ intracerebroventricularly injected into the right lateral ventricle of 8-weeks-old mice on a stereotaxic frame using a stainless steel cannula connected to a Hamilton syringe through a PE10 tube (stereotaxic coordinates in $\mathrm{mm}$ with reference to the bregma were $\mathrm{AP},-0.6$; $\mathrm{ML},-1.6$; DV, $-2.1)^{28}$. All mice were anesthetized with a gas mixture of $2 \%$ sevoflurane and $98 \%$ oxygen.

Usually, PC can be induced by toxic compounds used at $1 / 10$ of their toxic concentration (i.e., LPS, hypoxia or tMCAO $)^{29,30}$. Starting from this assumption, a concentration of L-BMAA ten times smaller than that used to mimic experimental ALS in vitro and in vivo, has been used.

\section{Tissue processing, immunostaining, and confocal immunofluorescence}

Immunostaining and confocal immunofluorescence procedures were performed as previously described ${ }^{31}$. Animals were anesthetized and transcardially perfused with saline solution containing $0.01 \mathrm{ml}$ heparin, followed by $4 \%$ paraformaldehyde in $0.1 \mathrm{~mol} / \mathrm{l} \mathrm{PBS}$ saline solution. Brains were rapidly removed on ice and postfixed overnight at $+4^{\circ} \mathrm{C}$ and cryoprotected in $30 \%$ sucrose in $0.1 \mathrm{M}$ phosphate buffer (PB) with sodium azide $0.02 \%$ for $24 \mathrm{~h}$ at $4^{\circ} \mathrm{C}$. Next, brains were sectioned frozen on a sliding cryostat at $40 \mu \mathrm{m}$ thickness, in rostrum-caudal direction. Afterwards, free floating serial sections were incubated with PB Triton X $0.3 \%$ and blocking solution $(0.5 \%$ milk, $10 \% \mathrm{FBS}, 1 \% \mathrm{BSA}$ ) for $1 \mathrm{~h}$ and $30 \mathrm{~min}$. The sections were incubated overnight at $+4{ }^{\circ} \mathrm{C}$ with the following primary antibodies: anti-NeuN (mouse monoclonal antibody; 1:500; Millipore, Milan, Italy), anti-NCX3 (1:3000; Swant, Bellinzona, Switzerland), anti-Glial Fibrillary Acidic protein (GFAP, rabbit polyclonal antibody; 1:500; Abcam, Cambridge, UK) and anti-ionized calcium binding adaptor molecule 1 (Iba1, rabbit polyclonal antibody; 1:500; Wako Diagnostic, Waco, VA, USA)

The sections were then incubated with the corresponding florescent-labeled secondary antibodies, Alexa 488/Alexa 594 conjugated antimouse/antirabbit IgGs
(Molecular Probes, Invitrogen S.R.L., Milan, Italy). Nuclei were counterstained with Hoechst (Sigma-Aldrich, Milan, Italy). Images were observed using a Zeiss LSM700 META/laser scanning confocal microscope (Zeiss, Oberkochen, Germany). Single images were taken with an optical thickness of $0.7 \mathrm{~m}$ and a resolution of $1024 \times 1024$. In double-labeled sections, the pattern of immune reactivity for both antigens was identical to that seen in singlestained material. Control double-immunofluorescence staining entailed the replacement of the primary antisera with normal serum (data not shown). To minimize a possible cross-reactivity between IgGs in double immunolabeling experiments, the full complement of secondary antibodies was maintained but the primary antisera were replaced with normal serum or only one primary antibody was applied (data not shown). In addition, the secondary antibodies were highly preadsorbed to the IgGs of numerous species. Tissue labeling without primary antibodies was also tested to exclude autofluorescence. No specific staining was observed under these control conditions, thus confirming the specificity of the immunosignals.

Quantification of GFAP, NCX3, and Iba1 fluorescence intensity on tissue sections at the level of the spinal cord, was quantified in terms of pixel intensity value by using the NIH image software, as described previously ${ }^{17,18}$. Briefly, digital images were taken with $\times 40$ or $\times 10$ objective and identical laser power settings and exposure times were applied to all the photographs from each experimental set. Images from the same areas of each brain region were compared. Results were expressed in arbitrary units. Three sections from each mouse were analyzed, with $n=3$ mice per treatment group.

To obtain an indirect measure of the amount of NCX3 in neurons, image analysis of NeuN was performed by $\mathrm{NIH}$ image software by measuring the intensity of fluorescent NCX3 immunolabeling in $50 \mathrm{NeuN}$ positive neurons for each group. The intensity of NCX3 immunoreactivity was expressed in arbitrary units.

Standard Nissl staining was employed on coronal step serial sections from spinal cord and brain stem. 


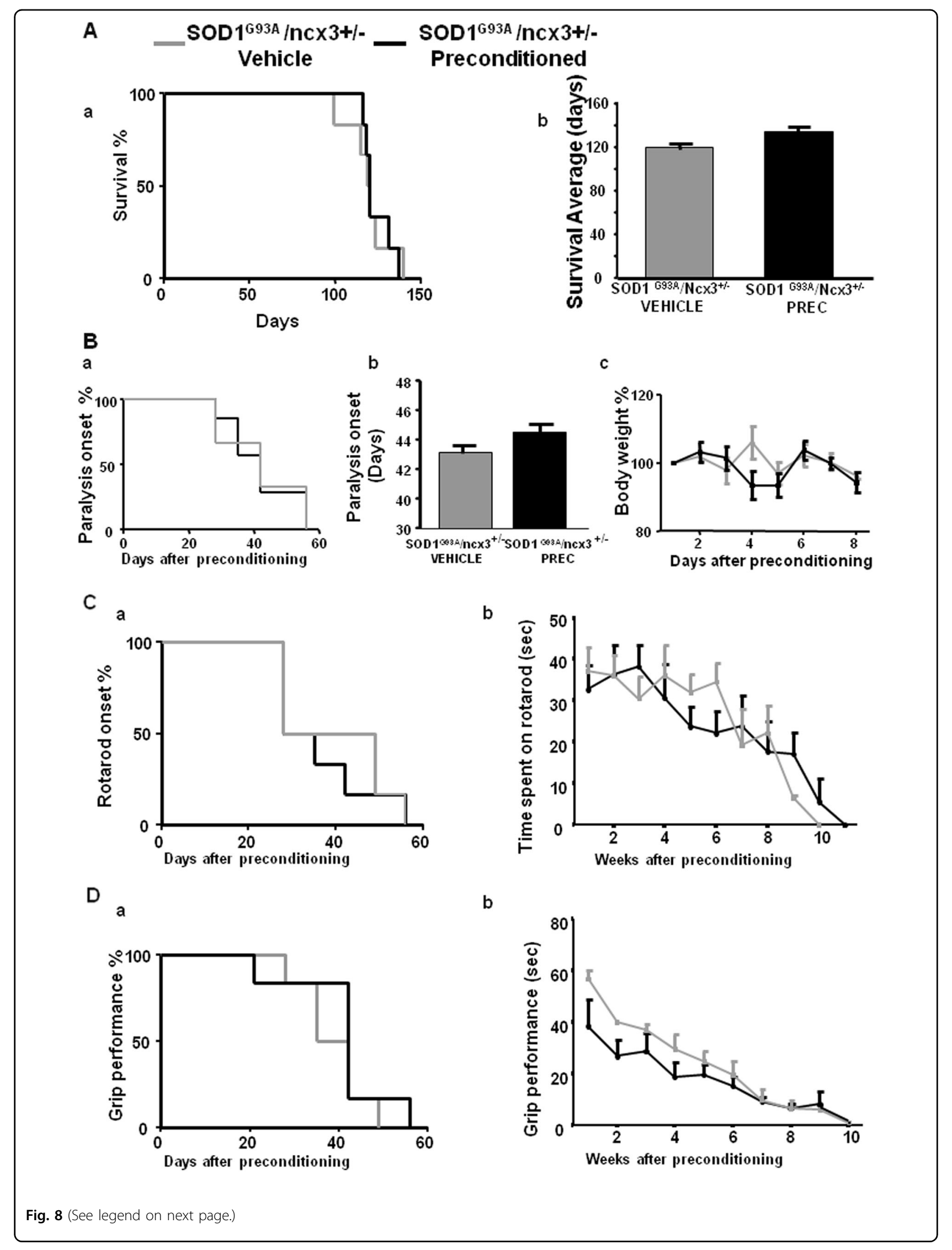




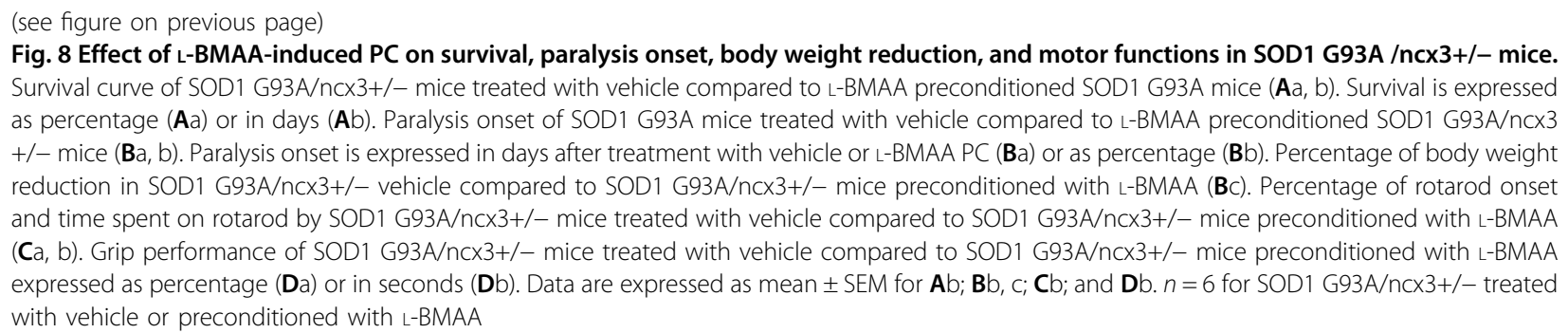

\section{Neuromuscolar junction (NMJ) analysis}

Gastrocnemius muscle was removed and snap-frozen in liquid nitrogen-cooled isopentane. To study the NMJ, gastrocnemius muscle, sectioned in $10 \mu \mathrm{m}$ thickness, in rostrum-caudal direction, was stained with a postsynaptic marker, $\alpha$-Bungarotoxin, Alexa 488 conjugate (1:500), and a pre-synaptic marker, Synaptophysin (rabbit polyclonal antibody 1:500; Abcam, Cambridge, UK). NM)s were scored according to whether there was complete co-localization of pre- and post-synaptic markers (fully innervated), partial co-localization (intermediate innervation), or only post-synaptic labeling (fully denervated). All of the NMJ analyses were performed on 270 or more NMJs from each group ${ }^{32}$.

\section{Motor neurons counting analysis}

Motor neurons were counted in the cervical spinal cord and in the brain stem facialis nucleus. Sections of each area were analyzed as previously described ${ }^{33}$. Frozen brain tissue and spinal cord were sectioned on a sliding cryostat at $20 \mu \mathrm{m}$, in rostrum-caudal direction. Four mice for each genotype and four slides from every mouse were analyzed.

Analyses were performed using image $J$ software in Polygonal-shaped neurons larger than $20 \mu \mathrm{m}$ with a welldefined cytoplasm, nucleus, and nucleolus ${ }^{34}$.

\section{Western blot analysis}

Western blot analysis was performed as previously described $^{30}$. Spinal cord, motor cortex and brain stem tissues were lysed in lysis buffer containing $50 \mathrm{mM}$ Tris- $\mathrm{HCl}, \mathrm{pH}$ 7.4, $150 \mathrm{mM} \mathrm{NaCl}, 1 \mathrm{mM}$ EDTA, 1\% Triton $\mathrm{X}-100$, and protease and phosphatase inhibitors. Samples were subjected to SDS-polyacrylamide gel electrophoresis (SDS-PAGE) and immunoblotted with specific antibodies. Polyclonal antibodies were used against NCX3 (rabbit polyclonal; 1:2000; Swant, Bellinzona, Switzerland), anti$\beta$-Actin (rabbit polyclonal 1:1000 dilution; Sigma-Aldrich, St. Louis, MO, USA). Immunoreactive bands were detected using ECL (GE Healthcare, Milan, Italy). The optical density of the bands (normalized for $\beta$-actin) was determined by Chemi-Doc Imaging System (Bio-Rad, Segrate, Italy $)^{35}$.

\section{RT-PCR experiments}

Tissues were quickly removed from mice, then immediately frozen on dry ice and stored at $-80^{\circ} \mathrm{C}$ until use. Total RNA was extracted with Trizol reagents, following supplier's instructions (Life Technologies, Monza, Italy). The first-strand cDNA was synthesized with $2 \mu \mathrm{g}$ of total RNA using the High Capacity cDNA Reverse Transcription Kit following supplier's instruction (Life Technologies, Monza, Italy). Quantitative real-time PCR with TaqMan assays for NCX3 gene and glucuronidase beta (Gusb) as housekeeping were performed in a 7500 realtime PCR system (Life Technologies, Monza, Italy). Samples were amplified simultaneously in triplicate in 1 assay run. Changes in mRNA levels were determined as the difference in threshold cycle $(\Delta \mathrm{Ct})$ between the target gene and the reference gene ${ }^{36}$.

\section{Purified synaptosomal preparation and $\left[\mathrm{Ca}^{2+}\right]_{\mathrm{i}}$ imaging}

Spinal cord synaptosomes were purified on discontinuous Percoll gradients, as previously described ${ }^{37}$. Briefly, tissues were homogenized in a medium containing $0.32 \mathrm{M}$ sucrose, $1 \mathrm{mM}$ EDTA, and $0.25 \mathrm{mM}$ DL-dithiothreitol ( $\mathrm{pH}$ 7.4). Each homogenate was centrifuged at $1000 \times g$ for $10 \mathrm{~min}$ at $4{ }^{\circ} \mathrm{C}$ and the supernatant was diluted at $14 \mathrm{ml} / \mathrm{g}$ with sucrose medium (pH 7.4). Two $\mathrm{ml}$ of the suspension were placed onto $8 \mathrm{ml}$ Percoll discontinuous gradient containing $0.32 \mathrm{M}$ sucrose and $3 \%, 10 \%, 15 \%$, and $23 \%$ Percoll ( $\mathrm{pH}$ 7.4). After centrifugation at 32,000 $\times g$ for $15 \mathrm{~min}$ at $4{ }^{\circ} \mathrm{C}$, synaptosomes were recovered between the $15 \%$ and $23 \%$ Percoll bands, diluted five times with HEPES buffer medium containing (in $\mathrm{mM}$ ): $125 \mathrm{NaCl}, 2.5 \mathrm{KCl}, 5$ $\mathrm{NaHCO}_{3}, 1.2 \mathrm{NaH}_{2} \mathrm{PO}_{4}, 1.2 \mathrm{MgSO}_{4}, 6$ glucose, and 25 HEPES ( $\mathrm{pH} 7.4$ ), and centrifuged at $15,000 \times \mathrm{g}$ for $15 \mathrm{~min}$ at $4{ }^{\circ} \mathrm{C}$. Finally, the pellet was resuspended in $1 \mathrm{ml}$ of medium B (145 mM NaCl, $3 \mathrm{mM} \mathrm{KCl}, 1.2 \mathrm{mM} \mathrm{MgCl}_{2}, 10$ $\mathrm{mM}$ glucose, and $10 \mathrm{mM}$ HEPES, $\mathrm{pH}$ 7.4) and stored on ice. Protein content was determined by the Bradford 
method $^{38}$. Percoll-purified synaptosomes were resuspended in medium $B(1 \mathrm{mg} / \mathrm{ml})$ and loaded with the ratiometric fluorescent $\mathrm{Ca}^{2+}$ indicator Fura-2 AM $(6 \mu \mathrm{M})^{12}$ in the presence of $16 \mu \mathrm{M}$ bovine serum albumin for $45 \mathrm{~min}$ at $37^{\circ} \mathrm{C}$. Dye-loaded synaptosomes were then washed by centrifugation, resuspended in medium $\mathrm{B}$ containing $1.2 \mathrm{mM} \mathrm{CaCl}_{2}$, and attached to poly-D-lysinecoated coverslips for $20 \mathrm{~min}$ at $37^{\circ} \mathrm{C}$. The coverslips were placed into a perfusion chamber (Medical System, Greenvale, NY, USA) mounted on the stage of an inverted Zeiss Axiovert 200 fluorescence microscope (Zeiss, Oberkochen, Germany) equipped with a $\times 40$ oil objective lens. Experiments were carried out with a digital imaging system composed of MicroMax 512BFT cooled CCD camera (Princeton Instruments, Trenton, NJ, USA), LAMBDA 10-2 filter wheel (Sutter Instruments, Novato, CA, USA), and META-MORPH/METAFLUOR Imaging System software (Universal Imaging, West Chester, PA, USA). Synaptosomes were illuminated at 340 and $380 \mathrm{~nm}$ wavelength by a $100-\mathrm{W}$ Xenon lamp (Osram, Berlin, Germany). The emitted light was passed through a 512 $\mathrm{nm}$ barrier filter. Images were digitized and analyzed using metafluor Imaging software. NCX activity was evaluated as $\mathrm{Ca}^{2+}$ uptake through the reverse mode by switching the normal Krebs medium to $\mathrm{Na}^{+}$-deficient $\mathrm{NMDG}^{+}$medium named $\mathrm{Na}^{+}$-free, containing (in $\mathrm{mM}$ ): $5.5 \mathrm{KCl}, 147 \mathrm{NMDG}, 1.2 \mathrm{MgCl}_{2}, 1.5 \mathrm{CaCl}_{2}, 10$ glucose, and 10 Hepes-Trizma (pH 7.4).

\section{Evaluation of motor performance}

Hindlimb grip test was conducted by placing the mouse on a grid ( $45 \mathrm{~cm}$ long $\times 28 \mathrm{~cm}$ large) upside-down $(30 \mathrm{~cm}$ above a foam pad). The test was performed once a week and the latency to fall off the grid was also measured up to a maximum of $60 \mathrm{~s}$.

Motor coordination and balance was assessed using a five-station mouse rotarod apparatus (Ugo Basile; Milan, Italy). In each station, the rod was $6 \mathrm{~cm}$ in length and $3 \mathrm{~cm}$ in diameter. Mice were trained to maintain balance at increasing speed up to a constant speed of $14 \mathrm{rpm}$ for three consecutive trials. The test sessions were conducted by one rotarod trial administered once a week. In this session, the speed of rotation was increased from 4 to 14 rpm over $60 \mathrm{~s}$. Mice had three trials on the rod, and the latencies to fall were measured once a week and then averaged. The maximum latency of $60 \mathrm{~s}$ was assigned to the mice that did not fall at all ${ }^{39}$.

Weekly evaluation of hindlimb paralysis was performed. Hindlimb paralysis was scored when the animal dragged one of its hindlimbs, and paralysis of a forelimb was scored when the mouse failed to use its forelimbs for walking or righting.
Body weight was measured immediately before each session of behavioral tests.

Disease endstage was defined by the inability of mice to right themselves within $20 \mathrm{~s}$ when placed on their sides.

\section{Statistical analysis}

Data were evaluated as means \pm SEM. Statistically significant differences among means were determined by one-way ANOVA followed by Student-Newman-Keuls post-hoc test for western blotting, cell counting, and realtime PCR analysis. Two-way ANOVA followed by Bonferroni post-hoc was used for motor performances test and body weight analysis. The Kaplan-Meier plot was used to evaluate survival, grip, rotarod and paralysis onset. Student's $t$-test was used for two groups comparison. Statistical significance was accepted at the $95 \%$ confidence level $(P<0.05)$. Statistical analyses were performed by using GraphPad Prism 5.0 (La Jolla, CA, USA). All experiments were carried out in a blinded manner.

\section{Study approval}

Experiments were performed according to the international guidelines for animal research and approved by the Animal Care Committee of "Federico II" University of Naples, Italy and Ministry of Health, Italy. All efforts were made to minimize animal suffering and to reduce the number of animals used.

\section{Acknowledgements \\ This work was supported by the following grants: ConSLA from ARISLA to G.P., Programma Operativo Nazionale (PON_01602 and PON03PE_00146_1) from MIUR to L.A.; POR Campania FESR 2007-2013 FARMABIONET (B25C1300023007) to G.P.; POR Campania FESR 2007- 2013 OCKEY (B25C13000280007) to G.D.R.; POR Campania FESR 2007-2013 MOVIE (B25C1300024007) to L.A.}

\section{Author details}

IIRCCS SDN, Via Gianturco, 80131 Naples, Italy. ²Division of Pharmacology, Department Neuroscience, School of Medicine, Federico II University of Naples, Via Pansini, 5, 80131 Naples, Italy. ${ }^{3}$ Laboratoire de Pharmacodynamie et de Thérapeutique, Bâtiment GE, Faculté de Médecine, Université Libre de Bruxelles, route de Lennik 808, 1070 Bruxelles, Belgium. ${ }^{4}$ Università Politecnica delle Marche, Ancona, Italy

Conflict of interest

The authors declare that they have no conflict of interest.

\section{Publisher's note}

Springer Nature remains neutral with regard to jurisdictional claims in published maps and institutional affiliations.

Supplementary Information accompanies this paper at https://doi.org/ 10.1038/s41419-017-0227-9.

Received: 5 October 2017 Revised: 6 December 2017 Accepted: 12 December 2017

Published online: 12 February 2018 


\section{References}

1. Dirnagl, U., Becker, K. \& Meisel, A. Preconditioning and tolerance against cerebral ischaemia: from experimental strategies to clinical use. Lancet Neurol. 8, 398-412 (2009).

2. Stenzel-Poore, M. P. et al. Effect of ischaemic preconditioning on genomic response to cerebral ischaemia: similarity to neuroprotective strategies in hibernation and hypoxia-tolerant states. Lancet 362, 1028-1037 (2003).

3. Karamyan, V. T. \& Speth, R. C. Animal models of BMAA neurotoxicity: a critical review. Life Sci. 82, 233-246 (2008).

4. Delzor, A. et al. Searching for a link between the I-BMAA neurotoxin and amyotrophic lateral sclerosis: a study protocol of the French BMAALS programme. BMJ Open 4, e005528 (2014).

5. Delzor, A., Marin, B., Boumediene, F., Preux, P. M. \& Couratier, P. BMAALS: French national project searching for a link between amyotrophic lateral sclerosis and the neurotoxic amino acid I-BMAA. Amyotroph. Lateral Scler. Frontotemporal Degener. 15, 155-156 (2014).

6. Siklos, L. et al. Intracellular calcium parallels motoneuron degeneration in SOD1 mutant mice. J. Neuropathol. Exp. Neurol. 57, 571-587 (1998).

7. Grosskreutz, J., Van Den Bosch, L. \& Keller, B. U. Calcium dysregulation in amyotrophic lateral sclerosis. Cell Calcium 47, 165-174 (2010).

8. Ferrer, I. et al. Calbindin D-28k and parvalbumin immunoreactivity in the frontal cortex in patients with frontal lobe dementia of non-Alzheimer type associated with amyotrophic lateral sclerosis. J. Neurol. Neurosurg. Psychiatry 56, 257-261 (1993).

9. von Lewinski, F. \& Keller, B. U. Ca ${ }^{2+}$, mitochondria and selective motoneuron vulnerability: implications for ALS. Trends Neurosci. 28, 494-500 (2005).

10. Annunziato, L., Pignataro, G. \& Di Renzo, G. F. Pharmacology of brain Na+/Ca ${ }^{2+}$ exchanger: from molecular biology to therapeutic perspectives. Pharmacol. Rev. 56, 633-654 (2004).

11. Molinaro, P. et al. Neurounina-1, a novel compound that increases $\mathrm{Na}+/ \mathrm{Ca}^{2+}$ exchanger activity, effectively protects against stroke damage. Mol. Pharmacol. 83, 142-156 (2013).

12. Molinaro, P. et al. Neuronal NCX1 overexpression induces stroke resistance while knockout induces vulnerability via Akt. J. Cereb. Blood. Flow. Metab. 36, 1790-1803 (2016)

13. Pannaccione, A. et al. A new concept: Abeta1-42 generates a hyperfunctional proteolytic NCX3 fragment that delays caspase-12 activation and neuronal death. J. Neurosci. 32, 10609-10617 (2012).

14. Annunziato, L., Boscia, F. \& Pignataro, G. lonic transporter activity in astrocytes, microglia, and oligodendrocytes during brain ischemia. J. Cereb. Blood Flow Metab. 33, 969-982 (2013).

15. Annunziato, L. et al. ncx1, ncx2, and ncx3 gene product expression and function in neuronal anoxia and brain ischemia. Ann. N. Y. Acad. Sci. 1099, 413-426 (2007)

16. Sokolow, S. et al. Impaired neuromuscular transmission and skeletal muscle fiber necrosis in mice lacking Na/Ca exchanger 3. J. Clin. Invest. 113, 265-273 (2004).

17. Boscia, F. et al. Silencing or knocking out the $\mathrm{Na}(+) / \mathrm{Ca}(2+)$ exchanger-3 (NCX3) impairs oligodendrocyte differentiation. Cell Death Differ. 19, 562-572 (2012).

18. Casamassa, A. et al. Ncx3 gene ablation impairs oligodendrocyte precursor response and increases susceptibility to experimental autoimmune encephalomyelitis. Glia 64, 1124-1137 (2016).

19. Mollereau, B. et al. Adaptive preconditioning in neurological diseases - therapeutic insights from proteostatic perturbations. Brain Res. 1648(Pt B), 603-616 (2016)
20. Cuomo, O. et al. Sumoylation of LYS590 of NCX3 f-Loop by SUMO1 participates in brain neuroprotection induced by ischemic preconditioning. Stroke 47, 1085-1093 (2016).

21. Pignataro, G et al. NCX1 and NCX3: two new effectors of delayed preconditioning in brain ischemia. Neurobiol. Dis. 45, 616-623 (2012).

22. Valsecchi, $\mathrm{V}$. et al. NCX1 is a novel target gene for hypoxia-inducible factor-1 in ischemic brain preconditioning. Stroke 42, 754-763 (2011).

23. Pignataro, G., Scorziello, A., Di Renzo, G. \& Annunziato, L. Post-ischemic brain damage: effect of ischemic preconditioning and postconditioning and identification of potential candidates for stroke therapy. FEBS J. 276, 46-57 (2009).

24. Muhling, T., Duda, J., Weishaupt, J. H., Ludolph, A. C. \& Liss, B. Elevated mRNA levels of distinct mitochondrial and plasma membrane $\mathrm{Ca}(2+)$ transporters in individual hypoglossal motor neurons of endstage SOD1 transgenic mice. Front. Cell. Neurosci. 8, 353 (2014).

25. Jaiswal, M. K. Calcium, mitochondria, and the pathogenesis of ALS: the good the bad, and the ugly. Front. Cell. Neurosci. 7, 199 (2013).

26. Gurney, M. E. Transgenic-mouse model of amyotrophic lateral sclerosis. N. Engl. J. Med. 331, 1721-1722 (1994).

27. Stifanese, R. et al. Adaptive modifications in the calpain/calpastatin system in brain cells after persistent alteration in $\mathrm{Ca}^{2+}$ homeostasis. J. Biol. Chem. 285, 631-643 (2010).

28. Pignataro, G., Simon, R. P. \& Xiong, Z. G. Prolonged activation of ASIC1a and the time window for neuroprotection in cerebral ischaemia. Brain 130(Pt 1), 151-158 (2007)

29. Rosenzweig, H. L. et al. Endotoxin preconditioning prevents cellular inflammatory response during ischemic neuroprotection in mice. Stroke $\mathbf{3 5}$ 2576-2581 (2004).

30. Pignataro, G. et al. nNOS and p-ERK involvement in the neuroprotection exerted by remote postconditioning in rats subjected to transient middle cerebral artery occlusion. Neurobiol. Dis. 54, 105-114 (2013).

31. Anzilotti, S. et al. Genetic ablation of homeodomain-interacting protein kinase 2 selectively induces apoptosis of cerebellar Purkinje cells during adulthood and generates an ataxic-like phenotype. Cell Death Dis. 6, e2004 (2015).

32. Liu, Y. et al. Ubiquitin-synaptobrevin fusion protein causes degeneration of pre-synaptic motor terminals in mice. J. Neurosci. 35, 11514-11531 (2015).

33. Gargiulo, S. et al. Imaging of brain TSPO expression in a mouse model of amyotrophic lateral sclerosis with (18)F-DPA-714 and micro-PET/CT. Eur. J. Nucl. Med. Mol. Imaging 43, 1348-1359 (2016).

34. Apolloni, S., Fabbrizio, P., Amadio, S. \& Volonte, C. Actions of the antihistaminergic clemastine on pre-symptomatic SOD1 G93A mice ameliorate ALS disease progression. J. Neuroinflamm. 13, 191 (2016).

35. Guida, N. et al. Methylmercury upregulates RE-1 silencing transcription factor (REST) in SH-SY5Y cells and mouse cerebellum. Neurotoxicology 52, 89-97 (2016).

36. Valsecchi, V., Boido, M., De Amicis, E., Piras, A. \& Vercelli, A. Expression of muscle-specific MiRNA 206 in the progression of disease in a murine SMA model. PLOS ONE 10, e0128560 (2015).

37. Dunkley, P. R., Jarvie, P. E., Heath, J. W., Kidd, G. J. \& Rostas, J. A. A rapid method for isolation of synaptosomes on Percoll gradients. Brain Res. 372, 115-129 (1986).

38. Bradford, M. M. A rapid and sensitive method for the quantitation of microgram quantities of protein utilizing the principle of protein-dye binding. Anal. Biochem. 72, 248-254 (1976)

39. Giampa, C. et al. Inhibition of the striatal specific phosphodiesterase PDE10A ameliorates striatal and cortical pathology in R6/2 mouse model of Huntington's disease. PLoS ONE 5, e13417 (2010). 